\title{
PERIPHERAL NEURITIS ASSOCIATED WITH A PULMONARY LESION IN A PATIENT WITH SYPHILIS*
}

\author{
BY
}

\author{
G. H. KNIGHT AND W. FOWLER
}

From the Department of Venereal Diseases, General Hospital, Birmingham

The present interest in the association of peripheral neuritis with bronchial carcinoma prompts us to record a case in which peripheral neuritis accompanied a pulmonary lesion presumed to be of syphilitic origin.

\section{Case Report}

A married bricklayer, aged 56 years, attended a venereal diseases clinic on April 29, 1954, complaining of ill health of 12 months' duration which he attributed to syphilis. Why he was so certain of the diagnosis was not disclosed. There was no history of early syphilis or any other venereal infection, extra-marital coitus was denied, and there had been no marital coitus for 14 years. This last statement was confirmed by his wife. His previous health had been good.

History of Present Illness. - There had been a general increase of malaise and weakness, with anorexia, loss of weight, and occasional attacks of vomiting, not related to any particular article of diet, which occurred during meals. He did not complain of any chest symptoms, but on questioning admitted to having had a cough for some considerable time, though he could not remember if this had preceded the other symptoms. He began to have difficulty in walking 14 days before admission. There was no history of any febrile attacks, haemoptysis, pains, or paraesthesia, and he had had no treatment of any kind. He was a heavy cigarette smoker and drank alcohol in moderation, and, as far as could be ascertained, there were no industrial hazards to which the illness could be attributed.

Clinical Examination.-He was ill and anxious-looking and had obviously lost weight. He walked with a stoppage gait and was dyspnoeic on slight exertion. No vomiting attacks occurred during his stay in hospital. The cough was loose and frequent with about $5 \mathrm{oz}$. mucopurulent sputum daily. There was no genital scarring or other signs suggestive of syphilis on the skin or mucous membranes. Examination revealed an area of consolidation in the upper lobe of the right lung. The heart appeared normal, the systolic blood pressure was $150 \mathrm{~mm}$., and the diastolic $75 \mathrm{~mm}$. $\mathrm{Hg}$. No abnormality was detected in the abdomen. The cranial nerves were intact. The superficial and tendon reflexes including the knee and ankle jerks were normal. There was no loss of power in the upper limbs, trunk, or thighs. The muscles supplied by the common peroneal nerves were paralysed with consequent foot drop and flexion of the toes. Palpation along the nerves in their superficial course revealed no thickening or other abnormality. A number of observers verify that the sensory divisions of these nerves were intact. He was afebrile and weighed $144 \mathrm{lb}$.

\section{Investigations}

Radiology. $-X$-ray of chest (Fig. 1) shows an area of consolidation in the anterior and postero-lateral segments of the upper lobe of the right lung.

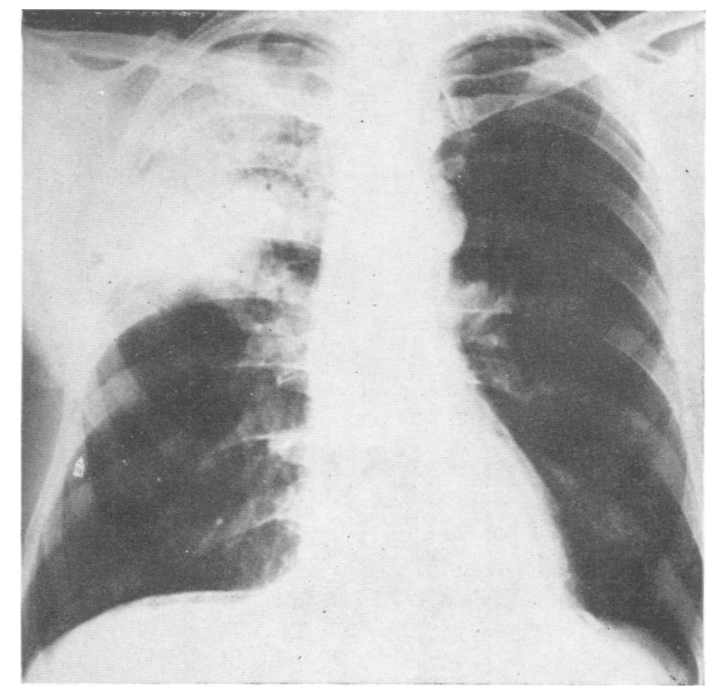

Fig. 1.- $X$-ray of patient, April 29, 1954, showing area of consolidation in anterior and postero-lateral segments of upper lobe of right lung.

Bronchoscopy (Mr. Victor Brookes).-No abnormality present.

Sputum.-Repeatedly negative for Mycobacterium tuberculosis and malignant cells.

Blood.-Haemoglobin $11.8 \mathrm{~g}$. (80 per cent.). Total white cell count 11,000 per ml. Differential count : neutrophil polymorphs 75 per cent., lymphocytes 22 per cent., eosinophils 0.5 per cent., monocytes 2 per cent. Erythrocyte sedimentation rate $85 \mathrm{~mm}$.

Serum Tests for Syphilis. - Wassermann reaction and Kahn test repeatedly positive. Quantitative Wassermann titre $1: 160$. 


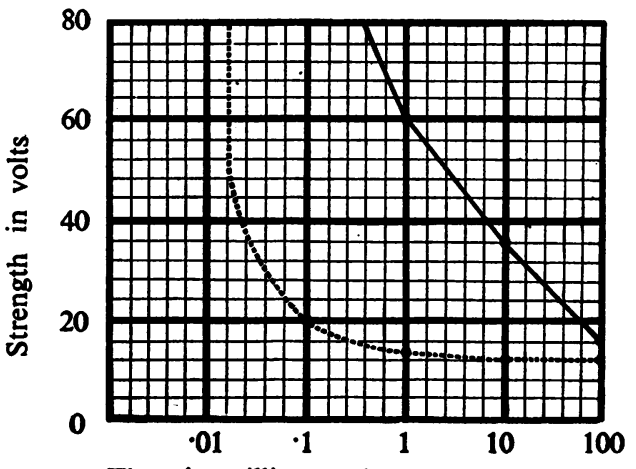

Time in milli-seconds
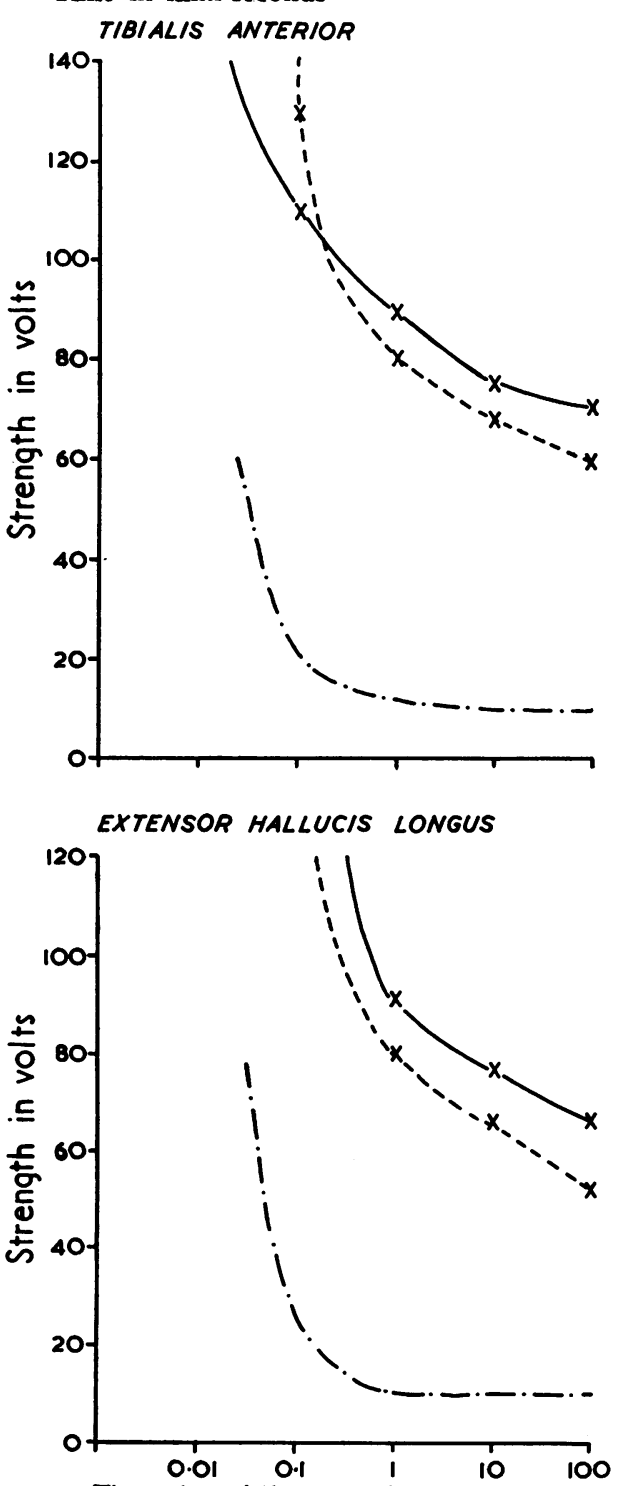

Time in milli-seconds

\section{- Denervated muscle \\ .......- Normal muscle}

Fig. 2.-Strength duration curve (control).

Cerebrospinal Fluid.-Wassermann reaction (Harrison-Wyler) complete inhibition of haemolysis in 2 vols. of cerebrospinal fluid, partial inhibition in 1 vol., complete haemolysis in remaining dilutions.

Urine.-Albumen a trace ; 2 polymorphs, scanty red cells, and a fair number of granular casts per high-power field.

Electrical Reaction of Muscles (Figs 2 and 3).These were greatly delayed.

Treatment.-30 gr. Potassium iodide three times daily and $8,750,000$ units crystalline penicillin were given

\section{EXTENSOR DIGITORUM LONGUS}
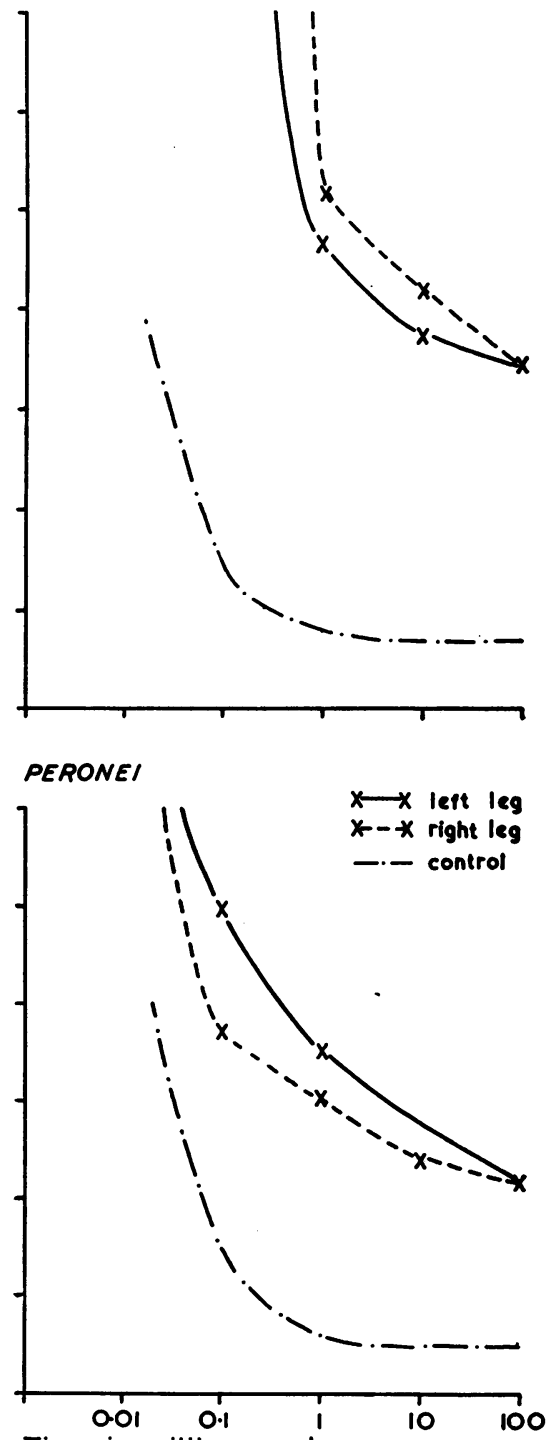

Time in milli-seconds 


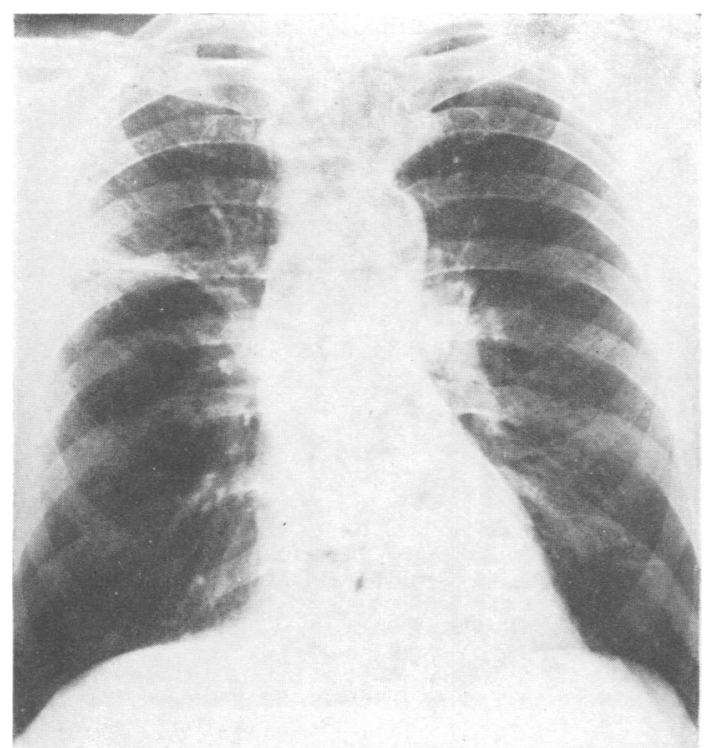

Fig. 4. $-X$-ray of patient, July 17,1954 , showing residual shadowing and slight elevation of upper horizontal fissure.

between May 8 and 26, 1954, followed by $1 \cdot 2$ g. N.A.B. and $6 \mathrm{ml}$. Bismostab (1.2 g. metallic bismuth).

Progress.-Towards the end of the second week of therapy the cough became less troublesome, the amount of sputum was greatly diminished, the abnormal lung signs were less pronounced, and the urine became normal. Subsequently the lung lesion steadily improved. Radiologically the shadow was appreciably smaller on May 25, 1954 ; on June 16 the lung was almost clear ; and on July 17 there was only residual shadowing and slight elevation of the superior horizontal fissure (Fig. 4). The general symptoms disappeared as the lung healed, the appetite returned gradually, and he began to put on weight. The erythrocyte sedimentation rate was $57 \mathrm{~mm}$. on June $2,28 \mathrm{~mm}$. on June 28 , and did not return to normal until August 17. On this date he weighed $154 \mathrm{lb}$., an increase of $10 \mathrm{lb}$. The quantitative Wassermann reaction was positive in a titre of $1: 80$ on August 17, 1954, and was unchanged on September 21, 1954, when Price's precipitation reaction (in a titre of $1: 32)$ and treponemal immobilization test were also positive. The signs of peripheral neuritis remained unchanged until 7 weeks after the commencement of therapy when power began to return to the affected muscles. Complete function was not restored until September 16, 1954.

\section{Discussion}

Syphilis of the lung is an uncommon pulmonary condition and presents no distinctive clinical or radiological features (Perry, 1952). In the absence of histological evidence, only a tentative diagnosis based upon the exclusion of commoner pulmonary conditions, particularly neoplasm and tuberculosis, is possible. A history of syphilis and unequivocal signs of this infection elsewhere in the body, positive serum tests, and response to antispecific therapy are additional guides to diagnosis (Hartung and Freedman, 1932). Similarly peripheral neuritis is rare in syphilis and may be indistinguishable clinically from that due to other causes (Hobhouse, 1945), although apparently at times the affected nerves may be thickened or show nodular swellings (Nonne, 1916).

The criteria for clinical diagnosis are similar to those for pulmonary syphilis: namely, positive anamnesis, absence of other aetiological factors, and a response to antisyphilitic therapy (Nonne, 1916).

In the present case there were no definite clinical signs of syphilis and no previous history, although the patient's choice of clinic might throw doubt on this. However, the persistence of high titre positive serum tests for syphilis almost 5 months after treatment had been started and 3 months after the lung lesion had healed suggests that the reactions were specific and not biologic false positives, as the latter tend to revert quickly to negative with cure of the infection responsible for their production. The positive TPI test confirms that the patient did in fact have syphilis.

There is nothing in the history of this case to suggest an independent aetiology for the peripheral neuritis, and the response to therapy suggests that there was some relationship between the pulmonary and nerve lesions. The response to therapy also showed that the pulmonary lesion was neither neoplasm, particularly of the bronchus, in which the incidence of peripheral neuritis is about 2 per cent. (Lennox and Prichard, 1950), nor tuberculosis, in which peripheral neuritis occasionally occurs (Wingfield, 1929) and makes peri-arteritis nodosa, a possible cause of the combined lesions, extremely unlikely. The rapidity with which the lung healed also makes a mycotic infection impossible. Though a coccal or viral origin for the pulmonary lesion cannot be eliminated with certainty, the long duration of the illness and the absence of any febrile episodes make either unlikely. We do not consider it unreasonable to assume that the pulmonary lesion was chronic because otherwise it is difficult to account for the ill health of a year's duration. Had treatment been initiated with metallic bismuth, it might have been possible to eliminate both these possible causes with certainty. Penicillin has too wide a range of activity to be of value as a therapeutic test; it was not given for this purpose, but to control the specific infection quickly so that the patient might be more able to 
cope with the more strenuous treatment which at that time it seemed probable he would have to undergo.

We therefore believe that a diagnosis of pulmonary syphilis is justifiable. From the rapidity with which the lung recovered and the completeness of this recovery it would appear that the lesion was an early one and probably confined to gummatous infiltration of the blood vessels of the peribronchial and alveolar tissues (Pearson and de Navasquez, 1938).

Having decided that the patient had syphilis and that this was probably responsible for the lung lesion, it remained to be seen if the peripheral neuritis could also be ascribed to the specific infection. This cannot be determined with any certainty, but the time at which the neuritis appeared and the slow recovery incline us to the opinion that the nerve lesion was not due to the specific infection but to a factor as yet unknown which causes peripheral neuritis in the late stages of some chronic infections, for example, bacillary dysentery, and probably also neuropathy in malignant diseases, especially of the bronchus.

\section{Summary}

A case is described with a lung lesion and peripheral neuritis. Evidence is produced that the patient had syphilis, and the progress of the case under treatment suggested that the lung lesion was of syphilitic origin. The peripheral neuritis was not thought to be specific.

\section{REFERENCES}

Hartung, A., and Freedman, J. (1932). J. Amer. med. Ass., 98, 1969. Hobhouse, N. (1945). Lancet, 2, 141.

Lennox, R., and Prichard S. (1950). Quart. J. Med., 19, 97.

Nonne, M. (1916). "Syphilis and the Nervous System", trans. C. R. Ball, 2nd ed., p. 234. Lippincott, Philadelphia.

Pearson, R. S. B., and de Navasquez, S. (1938). British Journal of Venereal Diseases, 14, 243.

Perry, K. M. A. (1952). In "Diseases of the Chest ", ed. G. Marshall and K. M. A. Perry. Vol. 2, p. 235. Butterworth, London.

Wingfield, R. C. (1929). “ A Text-Book of Pulmonary Tuberculosis for Students ", p. 170. Constable, London. 